\title{
ANÁLISIS DEL FONDO PARA EL DESARROLLO DEL EMPLEO INDUSTRIAL DE LA PROVINCIA DEL CHACO
}

DANIELA TORRENTE OMAR QUIRELLI

FACULTAD DE CIENCIAS ECONÓMICAS - UNNE 


\title{
RESUMEN
}

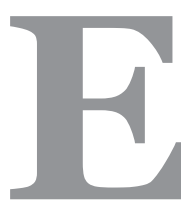

l presente trabajo toma como objeto de estudio el análisis de un ejemplo de política pública plasmada en una ley, a efectos de analizarla y determinar los alcances de la misma, sus posibles efectos sobre la actividad económica y los límites que enfrenta.

La medida analizada es el Fondo para el Desarrollo del Empleo Industrial, creado por Ley 6621 del año 2010 de la Provincia de Chaco, ley que fue reglamentada por el Decreto 956 de mayo del 2011 que creó el FODEI (Fondo para el Desarrollo del Empleo Industrial) y por la Resolución Ministerial 976 de Setiembre del 2011.

El trabajo se completa con el marco histórico y económico en el que se lleva a cabo la sanción de la ley.

El análisis y las conclusiones elaborados toman los conceptos y argumentos teóricos que permiten el estudio del diseño y la dinámica de las políticas públicas, así como, la importancia de su implementación efectiva para la concreción de determinados objetivos socioeconómicos.

Palabras Claves: Política pública - Industrialización - Provincia de Chaco.

\begin{abstract}
This paper takes as its object of analysis an example of public policy reflected into law, in order to analyze and determine meanings and effects of the measures on economic activity and limits to be faced.

The measure analyzed is the Global Industrial Employment Development, created by Law 6621 of 2010 of the Province of Chaco, and regulated by Decree 956 of May 2011 that created the FODEI (Fondo para el Desarrollo del Empleo Industrial) and Ministry Resolution 976 of September 2011.

The work is complemented by historical and economic context in which law is created.

The analysis and conclusions take concepts and theoretical arguments that allow the study of design and dynamics of public policy and the importance of effective implementation for the realization of certain socioeconomic objectives.
\end{abstract}

Keywords: Public policy - Industrialization - Province of Chaco. 


\section{INTRODUCCIÓN}

El objetivo del trabajo presentado es analizar el proceso de creación de una Política Pública y la gestión de la misma, encuadrándola dentro de los conceptos y argumentos que examinan el diseño y la dinámica de las políticas públicas, concentrándose en la fase de la implantación ${ }^{1}$ o implementación ${ }^{2}$, así como, la importancia de la adopción de un rol proactivo por parte del Estado en el apoyo al desarrollo de una determinada región.

La política estudiada es la ley 6621 que crea el Fondo para el Desarrollo del Empleo Industrial en la Provincia de Chaco.

La ley 6621, denominada Nueva Ley de Promoción Industrial, autoriza al Poder Ejecutivo Provincial a crear un Fondo Fiduciario para el Desarrollo Industrial. Tiene por objeto promover la inversión en proyectos industriales estratégicos a radicarse y radicados en la provincia, priorizando aquellos que contribuyan a la integración de cadenas de valor y/o se localicen en áreas prioritarias para el desarrollo provincial, otorgando financiamiento de largo plazo, subsidiando tasas de interés de préstamos y devolución total o parcial del impuesto a las ganancias para las utilidades reinvertidas.

Los temas desarrollados en el trabajo son:

1. Marco conceptual.

2. Caso de análisis: Ley 6621 del año 2010.

2.1 Contextualización histórico económica de la medida.

2.2 Breve análisis de la ley y de sus particularidades.

2.3 Identificación de los beneficios macroeconómicos y sociales a alcanzar.

3. Distintos aspectos asociados a la implementación de la Política Pública.

4. Conclusiones y recomendaciones.

\section{Marco conceptual}

\subsection{Política Pública, definiciones importantes}

Se puede definir a la política, como un programa de acción puesto en marcha por una decisión motivada en la razón de que algo no funciona en la realidad, y el carácter de pública, hace referencia a la intervención del gobierno y autoridades, lo que le da legitimidad y una naturaleza imperativa, puesto que emana de una autoridad que le confiere derecho a hacerlo.

Sumando ambos elementos y completando la definición, política pública, es un programa de acción que ordena la vida de una comunidad en torno a sus problemas, que opera en el plano económico, y tiene impacto sobre la vida de esa sociedad toda, en la que intervienen diferentes actores: grupos de presión, comités de expertos, gobierno, sectores beneficiarios de la medida, etc.

El carácter público de las políticas tiene una connotación que va mucho más allá de su contenido jurídico. Lo público está muy lejos de significar exclusividad estatal, significa el encuentro de la racionalidad estatal con la voluntad social. Hace referencia al gobierno y desde un contexto de pluralidad amplia (Cabrero Mendoza, E., 2000).

${ }^{1}$ Término acuñado por Tamayo Sáez.

2 Término acuñado por Roth. 
La presencia de los diferentes actores que participan en la elaboración de una medida de política pública es relevante, puesto que la definición de la misma implica el encuentro y consenso del sector público con la esfera privada y las entidades intermedias, a efectos de definir medidas que involucren la participación de los diferentes sectores implicados, de modo de asegurar la concreción de resultados y de establecer líneas de acción coherentes con las dificultades a las que se intenta dar solución.

Otra particularidad en la definición de cuestiones implicadas en la solución de un problema o de un conflicto de intereses es que siempre entra la realidad y las ideas conceptuales de quien(es) elabora(n) el programa. Aspecto que refuerza la importancia de la pluralidad de sectores involucrados en la definición de medidas que los afectan.

\subsection{El proceso de elaboración de una política pública, sus etapas}

El proceso de generación de políticas públicas comienza en la definición de un problema y finaliza en la evaluación de la puesta en marcha para resolver ese problema.

El proceso de las políticas públicas es asimilable conceptualmente a la noción de sistema. De tal forma que ninguna fase aislada del proceso tiene sentido en sí misma, ni basta para definir las características del proceso. Es perfectamente posible hacer una adecuada definición del problema determinando, a quién afecta la necesidad de afrontarlo, el momento adecuado para abordarlo y su posible coste, y sin embargo, no acertar con el plan de implantación de las soluciones que se han elegido para neutralizar y responder al problema (Tamayo Sáez, M., 1997).

Tener presente la unidad de la política pública y, por lo tanto, del proceso, no exime de la contemplación específica y detenida de cada una de las fases y elementos del proceso. Por el contrario, la realidad de la actuación administrativa pública se concentra generalmente en partes del proceso y no en la contemplación global de la política. Se podría argumentar con tranquilidad que la unidad de la política pertenece más bien al plano conceptual de los analistas de la política que al operativo, el de los responsables de cualquiera de las fases del proceso de la política pública (Tamayo Sáez, M., citando a Hogwood y Gunn).

Entender a la política pública como sistema, involucra diferentes cuestiones de interés en la puesta en marcha de planes que buscan lograr objetivos de mejoras de la situación de uno o varios sectores que forman parte de la esfera de actuación del gobierno. Por un lado, es necesario comprender que la puesta en marcha de una medida, sigue diferentes etapas, las cuáles son descriptas brevemente en los párrafos siguientes, pero, al mismo tiempo, la idea de sistema plantea que el logro de resultados deseados es multifactorial. La política pública es una unidad, un agregado, que depende no solo de un buen diagnóstico de un problema y un correcto diseño de la medida, sino que cada uno de los eslabones intervinientes en el proceso tiene que trabajar correctamente a efectos de contribuir al logro de resultados deseados.

Esta idea es importante, porque focaliza en la necesidad de que las medidas definidas surjan del consenso de los diferentes actores involucrados en el problema a ser abordado, pero, al mismo tiempo, lo establece como una condición necesaria pero insuficiente para el 
éxito de la misma; luego de creada la medida debe atravesar exitosamente una serie de etapas para asegurar el logro de los resultados deseados.

Las etapas que intervienen en el proceso de las políticas públicas son:

- Identificación y definición del problema.

- Formulación de alternativas.

- Adopción de una alternativa.

- Implantación de la alternativa seleccionada.

- Evaluación de los resultados obtenidos (Tamayo Sáez, M., 1997).

Las que en términos generales se describen de la siguiente manera:

La de identificación del problema, sus causas y la evaluación de la importancia de darle prioridad en la agenda, implica la definición de las dimensiones del problema, al igual que la proyección de cómo evolucionará si no se actúa sobre él.

Esta fase es continuada por la de incorporación de ese problema identificado a la agenda de prioridades del gobierno, la definición de objetivos y metas y por la búsqueda de soluciones en función a las alternativas disponibles y los costos y beneficios de cada una de ellas, al igual que la evaluación de cuál promete los mejores resultados con los menores efectos negativos.

Luego de haber analizado y determinado la viabilidad de la alternativa seleccionada, se adopta una decisión, lo que la convierte en una decisión de política pública y da lugar a la búsqueda y evaluación de viabilidad y apoyo legislativo para llevarla a la práctica.

Ejecución, implantación de la política. En esta etapa es importante la definición de quiénes son los responsables de la ejecución, así como los medios materiales para llevarla a cabo, y el necesario compromiso requerido a los funcionarios que intervienen en la puesta en marcha de la decisión tomada para asegurar la correcta gestión de los recursos involucrados y garantizar el trabajo en pos de los resultados deseados.

La evaluación implica la definición de mecanismos, indicadores y criterios objetivos, que permitan determinar si se han alcanzado los resultados esperados y, en función a ello, determinar si es conveniente continuar o no con la política. Si la decisión es continuar, se debe analizar la necesidad o no de realizar ajustes. La evaluación también implica la ponderación de si los resultados obtenidos han resultado justos.

\subsection{El rol de las políticas públicas en la concreción de resultados económicos y sociales}

Tal como se ha definido al comienzo del apartado, la política pública es un programa de acción puesto en marcha a raíz de una decisión gubernamental motivada en la razón de que algo no funciona en la realidad. Por lo tanto, se puede pensar en un conjunto de actividades debidamente programadas y consensuadas, incentivadas en la necesidad de corregir una situación que, de acuerdo a la agenda de prioridades de un gobierno, requiere ayuda del Estado para ser superada.

Si las medidas adoptadas buscan llevar a cabo modificaciones estructurales sobre una determinada realidad social o sobre el aparato productivo, es importante que dicha planificación 
se realice sobre la base de un diagnóstico correcto y muy profundo de las particularidades que caracterizan y afectan esa realidad.

En este sentido, es importante resaltar que paquetes de medidas que no incorporan estos elementos, propios de la idiosincrasia local y del contexto temporal en que son aplicados, pueden llevar al fracaso de las disposiciones adoptadas, aun cuando se trate de recomendaciones que hayan resultado exitosas en otros lugares o en otros tiempos.

En el plano económico-productivo, las políticas industrial, agropecuaria, comercial y financiera, desarrolladas con un fin estratégico, son útiles para contribuir a la corrección de desequilibrios en el mediano y en el largo plazo, aunque no todas las corrientes de las ciencias económicas coinciden en respaldar esta afirmación.

A diferencia de la corriente mayoritaria de la profesión que postula la necesidad de un sector público de pequeñas dimensiones que actúe de manera neutral en la economía, dedicándose solo a asegurar un adecuado manejo de los grandes agregados macroeconómicos, y cautelar el respeto de los derechos de la propiedad, la evidencia proveniente de países exitosos como Corea, Israel, Singapur, Nueva Zelanda o Irlanda parece indicar que el Estado está llamado a cumplir un rol más amplio, creando mercados e instituciones y apoyando el desarrollo de nuevas capacidades productivas y tecnológicas de una sociedad. Existen muchos y muy variados estilos de capitalismo en el mundo, pero en los países que mayor éxito están alcanzando en la actualidad, el sector público ha adoptado un rol proactivo tomando en sus manos la tarea de construir un modelo más vibrante y dinámico de capitalismo, basado en la creación y la incorporación de ventajas comparativas dinámicas originadas en el conocimiento. Fomentar la transformación estructural de largo plazo del aparato productivo, apoyando la implantación de industrias conocimiento intensivas, mejorar el acceso a la educación y su calidad, fomentar la capacitación de recursos humanos en la esfera del trabajo, profundizar el funcionamiento de los mercados de capitales, acelerar el acceso al mundo digital y a la Sociedad de la información de las firmas pequeñas y medianas y de los sectores de la sociedad hoy excluidos del nuevo "ambiente digital" en franca expansión, y velar por el acceso equitativo a bienes "meritorios" como salud, vivienda o seguridad social, parecen ser los ejes centrales sobre los que deberían construirse programas de política pública en las actuales circunstancias. Acciones de este tipo apuntan a mejorar simultáneamente productividad, equidad social y competitividad internacional (Katz, J., 2008).

Por lo tanto, la construcción de instituciones y capacidades tecnológicas y financieras que permitan potenciar el desarrollo productivo de una región, al tiempo que aseguren la mejora social, son clave para alcanzar objetivos de desarrollo. Pero este apoyo no puede ser indiscriminado, ni puede estar ausente la planificación; claramente, la naturaleza de escasez de los recursos y de falta de límites en las necesidades, lleva a la obligatoriedad de establecer prioridades en su asignación.

Al mismo tiempo, es indispensable pensar en términos de sustentabilidad, lo que implica que la estrategia de política pública bosquejada debe respetar tres condiciones básicas: ser económicamente viable, asegurar objetivos de distribución equitativa y ser compatible con el aseguramiento del resguardo del medio ambiente. 


\section{Caso de análisis: Ley 6621, del año 2010, que crea el Fondo para el Desarrollo del Empleo Industrial en la Provincia de Chaco}

En este apartado se analiza el caso concreto de una medida de política pública aplicada en la Provincia de Chaco. El análisis se completa con la incorporación de los elementos mencionados con anterioridad.

El apartado comienza con una breve introducción del contexto en el que fue implantada la medida, continua por la descripción de los principales aspectos a resaltar, finalizando con un análisis de las cuestiones destacables de acuerdo a los conceptos brevemente explicitados.

\subsection{Contextualización histórico económica de la medida. Importancia de la industrialización provincial.}

En el año 2003 en Argentina, con posterioridad a la salida de la convertibilidad, se comenzaron a visualizar los efectos de medidas adoptadas en 2002. La economía nacional creció a tasas del $8 \%$ promedio, hasta 2007 , disminuyendo este guarismo a partir de este año pero manteniendo tasas de crecimiento positivas.

La herencia recibida por el gobierno de Duhalde en 2002, fue un país desindustrializado y desarticulado económica y socialmente; con un marcado descreimiento en la clase dirigente.

La desindustrialización del país, que comenzó a mediados de los años 70 con las medidas de la última dictadura militar, cerró el círculo negativo con la suspensión de los beneficios promocionales a principios de los 90 y con el tipo de cambio no competitivo, proconsumo de bienes importados, característico de la década de los años 90.

En los años 90 la soja comenzó a destacarse como un producto líder en el flujo de comercio de commodities en los mercados mundiales, adquiriendo una importancia significativa en el proceso de transformación productiva de las economías productoras de productos primarios. Pero no solo la soja, también se experimentaron oportunidades de crecimiento basado en las exportaciones de otros productos primarios. La razón fue el mejoramiento de los términos del intercambio para los commodities. Entre las razones de este cambio a favor de los productos primarios se pueden mencionar el importante crecimiento de China e India, así como, la importancia destinada a los biocombustibles originada en la necesidad de encontrar fuentes energéticas alternativas al petróleo.

Estos cambios significaron un verdadero punto de inflexión para Argentina.

La salida tardía de la convertibilidad puso a la economía en situación de desventaja relativa, pero esta debilidad se transformó rápidamente en una fortaleza para la recuperación y el crecimiento del país hasta el año 2007.

En el plano de la política pública se generó una amplia gama de opciones, tanto económicas como sociales entre las que se pudo optar, con aciertos y errores, sin tener que hacer mayores concesiones; paradójicamente, ante la crisis tan profunda que había experimentado el país, las autoridades, apoyadas por el contexto internacional favorable, ampliaron los grados de libertad para hacer política económica y social en el país, los mismos grados de libertad 
que habían quedado tan reducidos en la década de los años 90 producto del corsé cambiario impuesto por la ley de convertibilidad.

El crecimiento experimentado por la economía del país se basó en el sostenimiento de un tipo de cambio real competitivo y estable, acompañado de una política monetaria acomodaticia consistente en la intervención en el mercado de divisas, que, temporalmente, permitieron potenciar los beneficios del contexto internacional favorable.

Acompañando y reforzando el crecimiento, se adoptaron medidas fiscales y redistributivas consistentes en una amplia gama de políticas de ingresos que impulsaron al consumo interno. Se pueden citar a modo de ejemplo, controles de precios de productos básicos, controles de salarios y tarifas de servicios públicos y productos energéticos, asignación universal por hijo, otorgamiento de jubilaciones y pensiones a personas sin aportes.

\subsubsection{La Provincia de Chaco en este contexto}

La Provincia del Chaco acompañó a la Argentina en su proceso de crecimiento económico ininterrumpido desde 2003. Durante la recesión mundial y sequía del año 2009, la actividad económica provincial fue apuntalada por la inversión pública en obras de infraestructura, retomando luego un sendero de crecimiento impulsado por el comercio exterior y la demanda del mercado interno (Prospecto letras Chaco, 2011).

La tasa de crecimiento para el periodo 2003-2010 en la Provincia de Chaco fue de aproximadamente el $78 \%$.

Este crecimiento fue a tasas estables e importantes, especialmente en los sectores productores de bienes, lo que hizo que la participación de los mismos en la estructura sectorial de la economía, pasara de un $22 \%$ promedio en la década de los años 90 a representar el $35 \%$ promedio en el periodo $2003-2010$.

Si bien el proceso de recuperación fue muy importante, se trató de un proceso asociado a las señales de precios que determina el mercado, manifestándose una extensión de la frontera agropecuaria y un importante crecimiento de la producción de soja y otros cultivos, motores de la recuperación de la actividad económica, lo que se verifica en el Producto Geográfico Bruto de la provincia y su desagregación por letras, en las que letra A, correspondiente a actividades tales como agricultura, ganadería, silvicultura y servicios para la producción primaria es determinante 3 .

Una debilidad de este proceso es que el predominio y protagonismo adquiridos por la producción primaria hace depender el crecimiento de políticas nacionales en materia cambiaria y comercial.

En adición a lo antes señalado se destacan como condicionantes de los resultados, la importancia que adquieren los precios de los commodities en los mercados mundiales y fenómenos climáticos. Por un lado, la producción primaria está ligada a la persistencia de condiciones favorables de precios, puesto que el rendimiento por hectárea de la provincia es inferior a las tierras de la Pampa Húmeda, entre otras razones porque la capa de materia orgánica del suelo

${ }^{3}$ Véase Ferreres, O (2011) estadísticas económicas. 
es también menor, lo que hace a la tierra menos productiva, al tiempo que, la mayor lejanía de los centros de exportación que tiene esta provincia encarece su transporte y la hace menos competitiva. Por otra parte se plantea la cuestión de las crisis asociadas a cambios climáticos, sequias e inundaciones que la mantienen altamente dependiente.

Estas cuestiones, entre otras, hacen que se ponga en duda la sostenibilidad del crecimiento a lo largo del tiempo.

En la presentación de letras para el financiamiento del gobierno provincial, a mediados del año 2011, se destacó la importancia de la soja en la generación de valor agregado provincial.

La actividad del sector primario históricamente se ha basado en el cultivo de algodón. La Provincia produce alrededor del 60\% del total nacional de este cultivo. En la última década, el avance del cultivo de soja ha sido destacado. Mientras que en la década del '9o la cosecha anual de la oleaginosa en la Provincia del Chaco oscilaba las 200000 toneladas, durante la campaña 2009/2010 esa cifra se ubicó por encima de 1500000 de toneladas. Según las estimaciones del Ministerio de Agricultura, Ganadería y Pesca (“MAGyP”), la cosecha 2010/2011 será aún mejor, con un avance de 6,7\% en las cantidades producidas. La participación de la Provincia del Chaco en la cosecha nacional de soja se ha incrementado sensiblemente: en la década del '9o oscilaba entre el $1 \%$ y $2 \%$, mientras que en la última campaña la contribución de la Provincia del Chaco a la producción de la oleaginosa alcanzó el 3\%...

...La expansión de este sector ha sido una importante fuente de dinamismo para la economía provincial dados los efectos de los ingresos por exportaciones sobre el gasto y el consumo interno (Prospecto letras Chaco)

Desde el año 2007, otra actividad muy dinámica en la provincia es la de la construcción ${ }^{4}$, la construcción pública es lo que más justifica el despegue de las tasas de crecimiento de Chaco con relación a la Nación a partir del año 2008.

Si bien la construcción privada también aumentó, y se vio beneficiada por el excedente de ahorros de la actividad primaria, lo que definió altas tasas de crecimiento, es la construcción de obras de infraestructura financiadas por el sector público, que se materializó en mejoras de apoyo a la producción.

El siguiente párrafo del libro El Chaco en Cifras, describe la magnitud de la obra pública en los últimos años.

Luego del estancamiento durante los 90 y primeros años del nuevo siglo, se están encarando acciones que demandarán varios años pero buscan poner al día la cobertura de necesidades de:

- Salud (refacción y construcción de hospitales, 6, y centros de salud) con inversiones previstas en el orden de los $\$ 350$ millones.

- Educación (refacción y construcción de 134 escuelas) con inversiones de \$300 millones.

- Se cuenta con un Plan Vial 2008-2015 para $400 \mathrm{~km}$ de rutas nuevas, 818 mantenidas y autovías Sáenz Peña-Resistencia y Resistencia-Formosa por \$2400 millones, y la autovía Resistencia-Makallé, con una inversión de \$400 millones en el año 2013. 
-También está prevista la mejora del Puerto de Barranqueras, con una inversión de \$230 millones en 5 años.

-El proyecto de electrificación total demandará \$3000 millones distribuidos del siguiente modo: Electroducto NEA- NOA \$1714 millones. Media y baja tensión \$1000 millones. Electrificación rural \$300 millones en 4 años.

-Acueductos por $\$ 2400$ millones

-Gasoductos tramo chaqueño por $\$ 5000$ millones.

-Hidrovía Paraná-Paraguay u\$s 112 millones para balizamiento, señalización y dragado.

-Fibra óptica doble anillado provincial más red troncal por un total de $\$ 173$ millones a finalizarse en mayo de 2012 (Barros, M. en Ferreres, O y otros. 2011 pp72).

Todavía quedan obras importantes pendientes, tales como la construcción del gasoducto, y el agua potable en algunas regiones de la provincia, pero se reactivó el ferrocarril Belgrano Cargas, se habilitó el puerto de Barranqueras, se produjeron importantes obras de electrificación rural, se mejoraron las rutas de acceso a localidades importantes de la provincia, además de la construcción de viviendas.

Resulta importante enfatizar el efecto de las obras de infraestructura, incluso en la corrección de desequilibrios regionales, puesto que la infraestructura en caminos, agua y energía eléctrica, es indispensable para mejorar las condiciones productivas de una provincia históricamente marginada.

A modo de síntesis, se puede destacar que el cambio de modelo económico Argentino post convertibilidad, con tipo de cambio real competitivo, permitió a la economía chaqueña desplegar parte del potencial de los sectores productores de bienes.

A ese primer impulso, se sumó el cambio de contexto mundial, con las importantes mejoras en los precios de los commodities en general y de los alimentos en particular, que se reflejan en la mejor relación de términos de intercambio del siglo para nuestro país. A estos factores exógenos positivos sobre la economía provincial, se sumaron como acción de los Estados Nacional y Provincial, por un lado una fuerte política de inversión pública en la provincia, fundamentalmente en infraestructura, financiada básicamente con recursos nacionales, que implican una significativa mejora de la potencialidad productiva de la provincia y activas políticas de ingresos tanto nacionales, como lo es la asignación universal por hijo, como provincial por otro, que reduce momentáneamente la pobreza e indigencia, y así lo confirman los indicadores estadísticos ${ }^{5}$, así como la desigualdad en la distribución del ingreso e impulsa el consumo, lo que permite mejoras en la evolución de la economía provincial, logrando tasas del crecimiento del PGB en los últimos años superiores a las nacionales.

En financiamiento y servicios para la producción, una característica de la provincia del Chaco es que ha mantenido la mayoría de participación estatal en canales de financiamiento, como lo es el Nuevo Banco del Chaco, además mantiene como activos de su propiedad las empresas proveedoras de agua y energía eléctrica, las cuales son, potencialmente, elementos

${ }^{5}$ Vease Ferreres O, 2011 Indicadores sociales. 
importantes en la planificación, puesto que pueden ser variables endógenas en la definición de un modelo de desarrollo para la provincia.

\subsubsection{Intento industrializador}

La Provincia de Chaco mantiene una asignatura pendiente con la actividad industrial; de hecho, la tasa de participación de la letra D, correspondiente a Industria en el PGB, cayó desde el año 2002 a 2009 de un 9.5\% de participación a un 8.3\%.

Pero, en la actualidad, se están desarrollando intentos industrializadores a través de mecanismos de incentivos a la generación de nuevas plantas y establecimientos industriales, así como al aumento del valor agregado. Para ello se adoptaron medidas de incentivo como las siguientes: a) Créditos con tasa subsidiada a través del Nuevo Banco del Chaco; b) Beneficios de Promoción Industrial - Ley 4.453; d) Subsidios de Contribuciones Patronales - Ley 6.093; y d) Fondo de Desarrollo del Empleo Industrial - Ley 6.621-.

Tal como se explicitó, la ley 6621, denominada nueva Ley de Promoción Industrial, constituye un intento concreto por generar un instrumento de política pública que contribuya a la generación de valor agregado industrial, asignatura pendiente en la provincia, que pertenece a la agenda de prioridades productivas para este distrito, en el apartado que se desarrolla a continuación se amplía el análisis de la misma.

\subsection{Breve análisis de la ley y de sus particularidades}

En la Provincia del Chaco, en el año 2010, fue sancionada por unanimidad de todos los bloques legislativos, la ley 6621, denominada Nueva Ley de Promoción Industrial, que autoriza al Poder Ejecutivo Provincial a crear un Fondo Fiduciario para el Desarrollo Industrial.

Dicha ley tiene por objeto promover la inversión en proyectos industriales estratégicos a radicarse y radicados en la provincia, priorizando aquellos que contribuyan a la integración de cadenas de valor y/o se localicen en áreas prioritarias para el desarrollo provincial, otorgando financiamiento de largo plazo, subsidiando tasas de interés de préstamos y devolución total o parcial del impuesto a las ganancias para las utilidades reinvertidas. Además, se autoriza al Ministerio de Economía Industria y Empleo a definir el listado de inversiones y áreas estratégicas que, a su juicio, reúnan los requisitos.

El Fondo Fiduciario se constituye con fondos contemplados en el presupuesto provincial. Para el año 2010 se estableció una suma de \$ 50 millones, y contempla que las leyes de presupuesto asignen idéntico monto por los próximos 10 años. Pero, el Poder Ejecutivo puede crear fideicomisos financieros u ordinarios con la finalidad de obtener recursos con destino al "Fondo para el Desarrollo del Empleo Industrial”. 


\subsubsection{Instrumentos de financiación y promoción a utilizarse según los establece la ley.}

Según los artículos 5 y 6 de la ley, los recursos del Fondo se aplicarán de la siguiente manera:

a) Préstamos de hasta diez años de plazo, con una tasa de interés nominal anual del siete por ciento (7\%) como mínimo, o en su defecto la tasa Badlar para bancos públicos, pudiendo expresar una variación entre 2 a 4 puntos en más o en menos. Hasta un 25\% del total del Fondo.

b) Subsidio de tasas de interés de préstamos acordados con entidades financieras públicas o privadas, el cual no podrá ser superior a diez puntos porcentuales. Hasta un $20 \%$.

c) Capitalización de empresas, a través de la adquisición, por parte del fondo, de acciones de las empresas interesadas, en la medida que las mismas constituyan una nueva emisión y cuyo producido se destine a financiar inversiones susceptibles de encuadrarse en los términos de la ley. Hasta un $15 \%$.

d) Devolución, total o parcial, del monto abonado en concepto de impuesto a las ganancias, cuando las utilidades que originaron dicho impuesto se destinen a su reinversión en bienes de capital o capital de trabajo, y del monto abonado en concepto de Fondo de Educación y Promoción Cooperativa (ley 23.427) para las cooperativas de 1er. y 2do grado, siempre que el destino de los recursos sea a los fines mencionados. Hasta un $20 \%$.

e) Financiamiento de todo otro instrumento de política industrial que el Poder Ejecutivo considere eficaz para el cumplimiento de sus objetivos, como la adquisición de terrenos con destino a la radicación de proyectos de inversión industrial. Hasta un 20\%.

Pudiendo la autoridad de aplicación, excepcionalmente, compensar entre los destinos referidos cuando existan remanentes de recursos en las asignaciones de algunos de los demás destinos, previo dictamen favorable de la Comisión de Seguimiento y Evaluación.

La respectiva ley fue reglamentada por el Decreto 956, de mayo del 2011, que crea el Fondo Fiduciario para el Desarrollo del Empleo Industrial (FODEI) y la Resolución 976 de setiembre del 2011 del Ministerio de Economía, Industria y Empleo crea la Unidad Técnica Ejecutora (UTE) que tendrá a su cargo la aplicación y puesta en marcha de la Ley.

\subsubsection{Criterios de asignación de los fondos}

La ley establece un criterio sectorial de asignación de los fondos, el cual se define en el artículo primero; éste es, dar preferencia especial al proceso industrial originado como consecuencia de la aplicación y/o utilización de las materias primas de origen agropecuario, y a otros sectores que el Poder Ejecutivo considere importantes a los fines del desarrollo productivo provincial. El Ministerio de Economía, Industria y Empleo, en su carácter de autoridad de aplicación, es quien tiene a cargo la confección de un listado con las inversiones y áreas que, a su juicio, reúnan los requisitos exigidos en dichos párrafos.

La ley también establece un criterio de orientación geográfica para los fondos, en el artículo 8; éste es, que el Poder Ejecutivo deberá considerar en el proceso de asignación de los 
recursos, las zonas o regiones geográficas en que se divide la provincia, de manera que la industrialización que se lleve a cabo sea armónica y equitativa en la jurisdicción provincial.

No obstante, dará especial preferencia a los proyectos de inversión que se localicen en el interior provincial. Este criterio de priorizar los proyectos ubicados en el interior provincial se refuerza en el artículo 10, en el que establece que la cantidad de ocupados para ser tomada como indicador de la devolución del impuesto a las ganancias será reducida en un 30\% para aquellas empresas ubicadas en el interior provincial. ${ }^{6}$

También establece el destino que las empresas deben dar a los fondos:

a) Destinar como mínimo un setenta por ciento (70\%) de las inversiones a la compra, construcción, fabricación, elaboración o importación definitiva de bienes de capital.

b) Mantener igual cantidad de trabajadores ocupados que en el ejercicio al que corresponden las utilidades gravadas. Si la empresa accede por más de un período a los beneficios de la ley, será requisito mantener o disminuir la ratio entre el valor contable de bienes de uso y las remuneraciones brutas totales en comparación con la misma ratio del ejercicio anterior.

\subsubsection{Aspectos vinculados a la creación de la ley':}

La sanción de esta ley fue la culminación de un proceso de búsqueda de consensos entre las cámaras empresarias, entidades intermedias, gremios, partidos políticos y autoridades del gobierno. La intención, según figura en el texto, es lograr soluciones al problema del retraso económico y social de la provincia, que muestra marcadas asimetrías con otras regiones del país, y se asienta en la concepción de que es el único camino para lograr un crecimiento sostenido y perdurable en el tiempo, con equidad social. Se pretende el desarrollo sustentable de los distintos sectores productivos que permitan la creación de empleo genuino, a la vez que logren dinamizar el mercado interno y generar un saldo exportable.

En esa búsqueda por lograr el desarrollo y luego de varias décadas de formar parte de las provincias con peores indicadores sociales y económicos, existe una visión compartida de las fuerzas de la producción y el trabajo de la importancia de las Políticas Públicas, a la luz de que por sí solo ningún sector industrial de la provincia cuenta con suficientes ventajas y escalas desde el punto de vista de las dotaciones factoriales y las ventajas comparativas para lograr el crecimiento sostenido.

\footnotetext{
$\overline{{ }^{6} \text { Esto es así porque la ley establece que el porcentaje de compensación del importe abonado en concepto de impuesto a las }}$ ganancias variará entre un cincuenta por ciento (a empresas con entre 101 y 150 ocupados) y un cien por ciento a empresas con menos 10 ocupados.

${ }^{7}$ Este apartado y el apartado 3 toman en consideración elementos aportados a través de la realización de entrevistas al ex Gerente Técnico del Consejo Económico Social de la Provincia de Chaco (CONES) Lic. Marcelo Nievas y del Presidente de la Unión Industrial de Chaco Lic. Francisco Lobera, ya que estas instituciones participaron activamente en la redacción del texto de la ley, y de los borradores previos de la misma.
} 


\subsection{Identificación de los beneficios macroeconómicos y sociales a alcanzar. Importancia de industrializar para lograr la sustentabilidad del crecimiento económico provincial}

Para la sustentabilidad del crecimiento, y su irreversibilidad y fortaleza ante cambios de contexto, sería importante profundizar en una estrategia que permita la radicación del valor agregado en la provincia y la generación de empleo local; en la que la presencia de una activa y efectiva interacción del Estado con el sector privado, consolide la industrialización de las materias primas para abastecer el consumo de la población chaqueña (sustitución de importaciones intra-provincia), al tiempo que permita lograr la consolidación de sectores productivos con el potencial de los recursos naturales, en escala suficiente para lograr excedentes exportables. De esta forma se podría consolidar un modelo económico-productivo, con crecientes inversiones privadas, donde la creación de empleo privado sea la principal herramienta para lograr mayor equidad distributiva perdurable en el tiempo.

Un breve análisis de las políticas económicas expansivas y redistributivas aplicadas por la Nación y por la Provincia permite concluir a priori que las mismas lograron, tal como se describe en apartados anteriores, mejoras en la actividad económica incrementando el ingreso de la población, impactando positivamente en el Consumo y en la Demanda Agregada de la Economía Provincial .

Las transferencias de la Nación a la Provincia de Chaco, a través de coparticipación de impuestos y de programas especiales, tanto sociales como de infraestructura, se ubican en niveles record históricos.

A partir del año 2000, con oscilaciones, se observa una tendencia en la composición del gasto público provincial consistente en la mayor incidencia de aquellos destinados a los servicios sociales. Es el caso de la función Educación, que en 2011 alcanzará el 34,5\% del gasto total (se cumple por primera vez el artículo 83 de la Constitución provincial que fija un piso de un 33\% de inversión educativa, superando el 13\% del PGB). Al mismo tiempo, la función salud superó el 4\% del PGB en los últimos años, participando en más del 11\% del gasto total, ubicándose así en los registros más altos de las dos últimas décadas (Barros, M. en Ferreres, O y otros. 2011 pp71).

Estas partidas, adicionadas a los desembolsos en obras de infraestructura y las transferencias a las familias, sumadas a una fuerte política de recomposición salarial a los empleados del sector publico provincial y a los jubilados de la caja previsional de la provincia, dieron lugar a una expansión del comercio y de la construcción residencial privada, situación potenciada por la alta propensión marginal al consumo de los sectores de ingresos medios y bajos, que son los beneficiarios de las políticas económicas. Como contrapartida, el nivel de gasto público provincial, plasmado en los presupuestos públicos de los últimos años, muestra incrementos superiores a la media nacional.

Partiendo del análisis del efecto multiplicador keynesiano de la políticas económicas, que gran parte de los alimentos, vestimentas, bienes durables, materiales de construcción, y todo tipo de bienes consumidos internamente se produzcan fuera del Chaco, generan una in- 
consistencia en el modelo de crecimiento económico a mediano plazo, toda vez que gran parte de los ingresos fiscales volcados al consumo no terminan generando valor agregado a radicarse localmente y empleo privado chaqueño.

En términos del modelo macroeconómico, la situación analizada se puede sintetizar diciendo que gran parte de las entradas compensatorias al flujo de la renta local, dada por las transferencias, los fondos recibidos del Estado Nacional y la producción primaria que se exporta fuera de las fronteras de la provincia, se filtran en concepto de importaciones de bienes y servicios (provenientes de otras provincias y de otros países), reduciendo significativamente el efecto multiplicador y reactivador de las políticas fiscales y de ingresos. Al mismo tiempo, este esquema de funcionamiento refuerza la dependencia provincial de ingresos recibidos de la nación y de los precios internacionales de los bienes que exporta, para mantener las tasas de crecimiento actuales.

De estos argumentos surge la necesidad de lograr la industrialización en la Provincia, que permita en una primera etapa satisfacer la demanda interna en bienes de consumo básico, y en una segunda etapa, generar saldos exportables de aquellos sectores que cuenten con potencialidades marcadas.

Entendemos que la correcta implantación de la Ley que crea el Fondo para el desarrollo del Empleo Industrial se constituye en un elemento importante para garantizar la industrialización de la Provincia y, de esa forma, hacer sostenible en el tiempo el crecimiento logrado en los últimos años. Al mismo tiempo, la generación de empleo privado formal, reducirá la previsión social sobre el gasto público del estado provincial, mejorará la recaudación impositiva y permitirá contar con financiamiento a nuevas políticas públicas.

\section{Distintos aspectos asociados a la implementación de la Política Pública:}

Lo establecido en el trabajo, nos conduce a aceptar que apoyar la industrialización es una prioridad de política pública que contribuirá al desarrollo económico y a la consolidación del crecimiento para los próximos años.

Partiendo de la idea de la pertinencia del beneficio creado, ahora pasaremos a reflexionar sobre las características del beneficio.

El análisis de la ley deja claro que la misma ha respetado las condiciones teóricas que se establecen para la creación de una política pública: la manera en que la misma fue redactada y la importancia dada a la reflexión sobre los mecanismos a ser desarrollados con participación multisectorial, nos asegura que se elaboró sobre la base de problemas concretos.

En la identificación del problema de la desindustrialización de la economía chaqueña y la importancia de darle prioridad a la solución, la Ley parte de un diagnóstico común de todos los actores políticos, sociales y económicos de la provincia: cámaras empresarias, confederación de trabajadores, estado provincial, partidos políticos, medios de prensa, etc.

La incorporación del problema a la agenda de prioridades del gobierno se sustenta en el consenso alcanzado por los actores políticos y económicos de la Provincia, lo que permitió in- 
corporar el problema en la agenda del gobierno en forma rápida y puso a trabajar a los equipos técnicos de los sectores, coordinados por el Consejo Económico y Social del Chaco (CONES) organismo creado por Ley y en el que se encuentran representados todos los sectores económicos, políticos y sociales.

Con relación a la adopción de la decisión, se resalta que la ley $\mathrm{N}^{\circ} 6211$ fue sancionada por unanimidad de todos los diputados, pasando por las distintas comisiones.

Lo que resulta clave analizar es si la manera en que está planteada la implantación, que es la etapa en la que se encuentra la ley, es adecuada para que la misma alcance sus objetivos, al tiempo que es interesante reflexionar sobre si los importes comprometidos resultarían suficientes y si los criterios de distribución de los fondos y de asignación geográfica, son coherentes con el objetivo sostenibilidad temporal del crecimiento. De igual manera ver los mecanismos de evaluación establecidos. A continuación, se reflexiona sobre estas cuestiones.

Aspectos relevantes asociadas a estas etapas ${ }^{9}$

1. Uno de los temas destacar, es la falta de definición de sectores a ser apoyados, lo que se desprende de la entrevista al presidente de la Unión Industrial; esa indefinición fue intencional, para evitar conflictos al interior de la institución, ya que la misma representa diferentes sectores de actividad industrial.

La ley establece que se dará prioridad a aquellos procesos industriales originados como consecuencia de la aplicación y/o utilización de las materias primas de origen agropecuario sin especificar criterios sectoriales concretos, sino más bien generales, para evitar conflictos en el seno de las cámaras empresariales (Federación Económica y Unión Industrial del Chaco), actores clave en el proceso de gestación. Tal como está planteado el proceso de asignación de fondos, existe la posibilidad de otorgar beneficios sin establecer verdaderos criterios de prioridades sectoriales, lo que podría hacer perder los objetivos inicialmente fijados; y de acuerdo a lo visto que se está realizando en la práctica, esto no está definido a nivel de gestión de los fondos, puesto que a diciembre de 2011 solo habían tres proyectos en evaluación y era una definición informal otorgar sumas no muy altas ${ }^{10}$ para llegar al mayor número posible de beneficiarios, lo que puede llevar a la dilución de los beneficios de la promoción.

Pero esta situación podría ser resuelta satisfactoriamente al definir el Ministerio de Economía la lista de sectores de actividad industrial prioritarios.

A pesar de ello, nos parece importante destacar que está en los objetivos del gobierno ${ }^{11}$, dar prioridad a los proyectos que generen agregación de valor a las materias primas producidas en la provincia, especialmente a los agroalimentos, y que creen empleo privado formal.

2. Con relación al importe global afectado al logro de los objetivos ( $\$ 50$ millones), es importante desatacar que, teniendo en cuenta el monto que representa el gasto público en la

\footnotetext{
${ }_{9}^{9}$ Para el análisis de esta etapa se incorpora al análisis, el resultado de una entrevista realizada el Lic. Alejandro Cucher, Subsecretario de Industria y Comercio del Ministerio de Economía Industria y Empleo de la Provincia de Chaco y responsable de la Unidad Técnica Ejecutora, al momento de la elaboración del trabajo.

${ }^{10}$ Está pensado otorgar préstamos de no más de $\$ 300.000$, y multisectorialmente.

${ }^{11}$ De acuerdo a lo especificado por el ex subsecretario de industria de la provincia, Alejandro Cucher
} 
provincia, una suma superior a 11000 millones de pesos, (8000 de presupuesto +3000 de transferencias + los importes destinados a obras públicas), no resulta un monto importante para el gobierno en términos relativos, el cual debería ajustarse teniendo en cuenta que está fijo en pesos y la inflación ha superado el $25 \%$ anual en la provincia y, si bien el texto de la ley establece que sea cuidado el poder adquisitivo de la suma comprometida, este importe no se ha actualizado entre 2010 y 2011.

3. Con relación al criterio de asignación regional, a priori, parecería acertado el estimular el desarrollo del interior de la provincia, pero siempre que el criterio esté asociado a la generación de valor agregado en la zona en la que se produce la materia prima o a algún criterio económico u objetivo que justifique la radicación en esa localización y que, una vez establecida, le permita subsistir sin el beneficio. Si el criterio responde a otras cuestiones, o a falta de planificación, no resulta razonable de ser establecido. Por lo general, en las proximidades a los grandes aglomerados, existe una infraestructura de servicios y de apoyo a la producción que sería importante aprovechar. Por ejemplo, el Departamento Libertad (Puerto Tirol) es un aglomerado industrial que podría ser óptimo potenciar en términos de valor agregado.

4. Con relación al criterio de asignación de los fondos en términos de porcentajes nos resulta importante destacar dos alternativas, la de subsidio de tasas de interés, porque permite incrementar el importe asignado, ya que impacta únicamente el monto del subsidio de tasas y no del capital prestado, al tiempo que transfiere el criterio de asignación y seguimiento a las entidades financieras, que tienen una mayor experiencia en evaluar la factibilidad de proyectos de inversión, más aun considerando que la provincia mantiene un banco público.

También nos parece importante destacar que una alternativa interesante es la de capitalización de empresas, a través de la adquisición, por parte del fondo, de acciones de las empresas interesadas porque permitiría impulsar proyectos nuevos con poco capital inicial, que no califiquen para asistencias crediticias y fundamentalmente que no puedan afrontar en los primeros años de vida el pago de servicios y capital de deuda.

5. Finalmente, se destaca lo siguiente, con respecto a la responsabilidad de la Ejecución de esta Política Pública el Decreto 956 del Gobernador de la Provincia faculta al Ministerio de Economía, en su carácter de autoridad de aplicación, a crear la Unidad Técnica Ejecutora (UTE) con el fin de administrar y evaluar los proyectos presentados. La misma estará integrada por el Ministro de Economía, el Subsecretario de Industria y un coordinador funcionario de la Subsecretaria. A su vez, la ley contempla la creación de la Comisión de Seguimiento y Evaluación encargada de verificar el cumplimiento de la Ley, que se reunirá con frecuencia trimestral para en un análisis ex post de los proyectos aprobados. Entendemos que es clave el eficiente funcionamiento de la UTE, tanto en tareas de difusión, capacitación, evaluación y seguimiento de los objetivos de la ley. 
Antes de pasar al apartado de conclusiones cabe aclarar que al momento de elaboración del trabajo, diciembre de 2011, prácticamente dos años después de sancionada la ley, solo habían tres presentaciones en evaluación y no había ningún desembolso efectuado, lo que hace poner en duda la efectiva implantación del beneficio, con el correspondiente desgaste y descreimiento que genera la falta de compromiso con la medida por parte de quienes deben gestionarla, en aquellos sectores que trabajaron para llevar a cabo la creación del beneficio.

\section{Conclusiones y recomendaciones:}

En lo que hace al proceso de creación de la Política Pública bajo estudio, se destacan, el grado de consenso que logró la determinación del problema, la incorporación de ese problema en la agenda política con la activa participación de todos los actores políticos, sociales y económicos de la provincia y la rápida adopción de la decisión de política pública a través del tratamiento y la aprobación de la ley.

Las experiencias sugieren que es clave disponer de variados mecanismos integradores internos que ayuden a la creación de reales comunidades de sentido. Bajo este marco cobran más importancia, por ejemplo, los planes elaborados participativamente entre todos los actores implicados en las acciones intersectoriales, ya que pueden contribuir a generar visiones compartidas... Es evidente además que cuando ella (la intersectorialidad) tiene un fundamento político, hay mayores probabilidades de que se venzan resistencias. De hecho un proyecto compartido en términos de políticas públicas proactivas para la mejora de la calidad de vida y la lucha contra la exclusión social, provee un marco no solo teórica sino políticamente afín con un enfoque intersectorial... Es pertinente, por tanto, reiterar el requerimiento que se suele hacer cuando se trata de introducir innovación: examinar antes el contexto socio político y la cultura política dominante, de modo de determinar cómo construir la viabilidad política del cambio propuesto (Cunill Grau, N, 2005).

Con relación a la implantación, podemos concluir que en términos generales, por los argumentos expuestos en el trabajo, nos parece importante tender al fortalecimiento de la actividad industrial local, con capitales locales y con una visión de un desarrollo integrado y equilibrado sectorialmente, pero dada la necesidad de gestionar los escasos recursos disponibles de manera adecuada para alcanzar estos objetivos, nos parece determinante la elaboración de una agenda de prioridades, en la asignación de los fondos.

Con relación a la necesidad de potenciar la generación de empleo genuino y de calidad, en términos de beneficios sociales, que implícitamente es uno de los argumentos sobre los que se asienta la ley, nos parece importante destacar que esto representa una continuidad en las medidas encaradas en los últimos años por los gobiernos nacional y provincial en materia social y económica, que se reforzó frente a la crisis financiera internacional.

“...las políticas adoptadas en la Argentina para hacer frente a la crisis se desplegaron en el periodo que va desde fines de 2008 a fines de 2009. Éstas abarcaron una diversidad de sectores y buscaron ampliar el papel del Estado y reconocer la importancia del trabajo como 
articulador de la inserción social. La mayoría tiene carácter transitorio y se orientan a reactivar la demanda y mantener los niveles de empleo ante la perspectiva de la reactivación de la economía. Sin embargo, coexisten con ellas algunas otras que implican cambios estructurales en la política social, abriendo una ventana de oportunidad a partir de la crisis que fue aprovechada por el gobierno para promover sus iniciativas....

Finalmente, en lo que respecta a infraestructura, se amplió el presupuesto disponible para obras públicas en 6925 millones de dólares. Estas inversiones se orientaron a infraestructura social y a infraestructura económica - productiva” (Repetto, F y Potenza del Masetto, F. 2011).

Políticas sociales proactivas, sumadas a políticas de generación de infraestructura productiva y política de creación y radicación de valor agregado en los distintos territorios que forman el país, nos parecen elementos indispensables para la superación de asimetrías regionales que presenta la Argentina, y en ese marco se inscribe la sanción de la Ley del Fondo para el Desarrollo del Empleo Industrial.

Sin embargo, vemos algunos aspectos que podrían permitir el éxito en la aplicación de la política y potenciar los efectos buscados con la misma:

- Establecer criterios objetivos de elegibilidad y priorización de los sectores productivos y regiones geográficas a favorecer, en función a la agregación de valor y la generación de empleo. Esto requerirá de un estudio de matriz productiva sectorial que permita obtener ratios de eficiencia económica y social en la aplicación del Fondo.

- Gestionar ante el Gobierno Nacional el aporte de una suma de dinero adicional prevista anualmente por la Provincia, como forma de compensar las asimetrías existentes entre distintas regiones y provincias del país. De esta forma, en principio se podrían incrementar los efectos económicos y sociales de política analizada.

- Introducir reformas en el funcionamiento y/o composición de la Unidad Técnica Ejecutora, establecer un mecanismo de gestión por resultados y la fijación de metas de cumplimiento cuantitativo y cualitativo. En este esquema, la función de la UTE no solo se limitará al análisis de proyectos presentados, sino a la difusión, capacitación, seguimiento, evaluación y medición de resultados de los proyectos.

Sostenemos que el atraso relativo de la Provincia del Chaco no se superará solamente con la aplicación de esta ley, sino con un conjunto de políticas públicas nacionales y provinciales que regeneren las condiciones para la inversión productiva. Sin embargo, la importancia estratégica que tiene la rápida y eficiente implementación de la misma, en términos de credibilidad en la gestión pública, es enorme, dado el consenso de todos actores políticos, sociales y económicos de la provincia sobre la necesidad contar con la Nueva Ley de Promoción Industrial.

Es importante resaltar la importancia de incentivar la generación de valor agregado industrial como fuente de afianzamiento del crecimiento económico y para que el mismo se derrame al resto de la sociedad; de esa manera se puede quitar presión al gobierno en su rol de interventor para redistribuir los resultados del crecimiento generado sobre las vertientes antes mencionadas. 
Tomar en cuenta la planificación regional sobre la base las fortalezas y debilidades de cada región del país sin propiciar la repetición de errores cometidos en el pasado, es crucial.

Finalmente, es altamente probable que este intento de la Provincia del Chaco por la industrialización de su economía y el fortalecimiento de las cadenas de valor, sea condición necesaria pero no suficiente parar reducir las asimetrías y lograr el desarrollo sustentable, ya que requieran el acompañamiento no solo del Gobierno Nacional con una visión realmente Federal en el armado de las Políticas Económicas, que contemple un firme compromiso con el desarrollo de las Economías Regionales, sino también de una visión compartida por la provincias de las región, con similares perfiles productivos y mismas necesidades, que articule acciones conjuntas del sector público y privado, relacionadas tanto con la concreción de obras de infraestructura estratégicas para dar sustento y continuidad a la potencialidad productiva de la región, como a la definición de una agenda de políticas públicas de largo plazo.

\section{REFERENCIAS BIBLIOGRÁFICAS}

Braun, M. y Llach ,L. (2010) Macroeconomía Argentina. $2^{\mathrm{a}}$ ed. Alfaomega. Buenos Aires. Argentina.

Cabrero Mendoza, E. (200o) Usos y costumbres en la hechura de las políticas públicas en México. Límite de las policy sciences en contextos cultural y políticamente diferentes, en Gestión y Política Pública, vol IX, 2.

Cunilll Grau, N. (2005) La intersectorialidad en el gobierno y la gestión de la política soical. X Congreso Internacional del CLAD sobre la reforma del estado y de la Administración Pública. Santiago de Chile. Chile.

Ferreres, O (2011) El Chaco. Su historia en cifras. Fundación Norte y Sur. CFI y Gobierno de la provincia de Chaco. Librería de la Paz. Resistencia Chaco.

Katz, J. (2008) Una nueva visita a la teoría del desarrollo económico. Copyright (CNaciones Unidas. Santiago de Chile.

Repetto, F. y Potenza Dal Masetto, F. (2011) Protección social en Argentina. CIP-PEC, CEPAL, Fundación Tzedaka y Universidad de San Andrés. Buenos Aires, Argentina.

Tamayo Sáez, M. (1997) El análisis de las políticas públicas. Alianza Editorial, Madrid. 


\section{CURRÍCULUM VITAE}

ESP. LIC. DANIELA TORRENTE.

Profesora Adjunta de Macroeconomía I. Investigadora Categoría IV.

dtorrente@eco.unne.edu.ar

LIC. OMAR QUIRELLI.

Profesor Adjunto de Macroeconomía I, Facultad de Ciencias Económicas de la UNNE. omarquirelli@arnet.com.ar 